Discussion Papers in

Economics and Econometrics

\begin{tabular}{||l||}
\hline THE DYNAMICS OF PUBLIC OPINION \\
UNDER MAJORITY RULES \\
Antonella lanni \\
and \\
Valentina Corradi \\
No. 0109 \\
\hline
\end{tabular}

This paper is available on our website http://www/soton.ac.uk/ econweb/dp/dp01.html 


\title{
The Dynamics of Public Opinion under Majority Rules*
}

\author{
Antonella Ianni ${ }^{\ddagger}$ \\ Valentina Corradi \\ University of Southampton (UK), University of Exeter (UK)
}

February, 2001

\begin{abstract}
This note explains the process of public opinion formation via a locally interactive, space-time analysis. The model we use is a special case of the general framework for modelling social interaction proposed in Blume and Durlauf (2001). In the reduced form of the model we study how each individual, when faced with the choice of one, out of two, opinions, tends to conform to the opinion held by the majority of her neighbours. We consider different, symmetric and asymmetric, majority rules. Depending on the specific behavioral rule, the aggregate process of opinion formation may display contagion on one specific opinion, or consensus among all individuals in the population, or co-existence of both opinions. Whenever consensus obtains, we observe the formation of homogeneous areas (clusters) that seem almost stationary along the dynamics.
\end{abstract}

\footnotetext{
${ }^{*}$ Keywords: Majority Rules, Public Opinion, Contagion, Co-existence, Clustering, Local Interaction.

${ }^{\dagger}$ The authors wish to thank A. Postelwaite and two anonymous referees for very helpful suggestions.

${ }^{\ddagger}$ Address for Correspondence: A. Ianni, Department of Economics, University of Southampton, Southampton SO17 1BJ, U.K.. e-mail: a.ianni@soton.ac.uk
} 


\section{Introduction}

Although scholars, observers and analysts do not seem to agree as to what public opinion actually is, there seems to be a widespread consensus that it should be studied as an "interactive, multidimensional, continuously changing phenomenon whose diverse aspects form causally interrelated patternings" (Crespi (1997), p. ix ${ }^{1}$ ). Two particular aspects are often emphasized in the sociological literature. The first is the fact that individuals faced with different choices as to whom - or what - to support show a tendency to be influenced by the opinion of some collective majority (mutual awareness, as defined in Crespi (1997)). The second is that environmental conditions that are specific to each agent seem to matter in determining the outcome of individual choices (situational correlates of opinion, as in Crespi (1997)). These features of the public opinion process seem to be well documented in terms of experimental and empirical evidence.

This note studies a simple dynamic model of public opinion formation and aims at explaining the stylized fact that the support for one, out of two, issues at stake often shows a high degree of local homogeneity and persistent cross-sectional variance, that is only partly explained by fundamental differences in economic conditions. An intuitive explanation of this stylized fact is that each individual may show a tendency to conform to the opinion of what (s)he perceives to be the opinion of a collective majority. Since communication is highly decentralized among a large group of individuals, each individual's perception of such majority may only be based on local features of the environment where interaction takes place. In particular, the opinion held within the small subset of an individual's neighbours, colleagues, friends or relatives, may be given a higher weight, then any information that is publicly available.

This note shows that the joint effect of local interaction and a tendency to agree with the opinion held by the majority, contribute to explain the creation of large homogeneous areas. The way this is done is by modeling the process of public opinion formation as a dynamic process, characterized by locally interactive patterns of behavior.

We postulate that the behaviour of each individual in forming an opinion is described by simple majority rules, that we take as primitives. Although we do not model it in detail, we show that these reduced forms are consistent with an explicit microeconomic foundation of the kind modeled in Blume and Durlauf (2000), Brock and Durlauf (2000, 2001), and Durlauf (1999) for general locally interactive settings. 
Along these lines, in Ianni and Corradi (2000) we study a simple model of preelectoral opinion formation that produces a reduced form consistent with majority rule behaviour.

We refer to the process of public opinion in its collective dimension as to the dynamic process generated by the aggregate of all the individual decisions, and we provide a stochastic formulation of its dynamics. In the paper we focus on a class of threshold functions and show how seemingly minor changes in the specification may have a relevant effect on the aggregate properties of the process. In fact, according to the specific behavioral rule followed by each individual, in the long run we may have contagion, where everyone will eventually agree on, say, opinion 1 , or consensus where everyone will eventually agree on either of the two opinions, or we may instead have coexistence of both opinions. However, a common feature which we observe along the dynamics is the formation of large homogeneous areas. In the particular case in which consensus occurs, we are actually able to study the rate at which these areas grow and explain why along the dynamics they seem almost stationary.

Issues related to the process of public opinion formation are not tangential to economic theory. Public opinion plays a key role in shaping animal spirits, patterns of consumer and producer behaviour expectations and in particular voting decisions. As for the latter aspect, the specifications we study in this paper are motivated in terms of band-wagon effect (voters favour the party that is doing well in the polls), or projection effect (voters tend to project their intended vote onto their election outcome expectations). Since the theoretical studies of Simon (1954) and Baumol (1957), empirical work carried out in the UK and in the USA seems to provide evidence for these hypothesis (see McAlister and Studlar (1991), Zuckerman A.S., Valentino and Zuckerman E.W. (1994) and therein references).

Henshel and Johnston (1987) provide an interesting survey of bandwagon theory, according to which there is a strong effect from opinion polls to voters decisions. Basically the fact that polls give an advantage to a candidate induces voters to switch their preference in favor of that candidate. This direct effect from polls to voters' opinion is mainly explained as a desire of conformity, both in the sense that people conform to what they believe is the proper norm and in the sense that people feel reassured in adopting the opinion held by the majority. Henshel and Johnston (1987) also point out an indirect effect: if polls give advantage to a candidate, more funds and endorsements will be directed to that candidate, by this increasing her/his share of support. Broadly speaking opinion polls can be interpreted as an indicator 
of the opinion held by the entire population. For example, via lab experiments, Nadeau, Cloutier and Guay (1987) provide evidence in favor of a bandwagon effect in opinion formation: in particular they find that individuals tend to switch their opinion towards the one expressed by the majority of the participants in the experiment.

As the bandwagon theory explains why each individual tends to conform to the majority, we should expect to observe growing consensus in an electoral campaign. However such theory does not allow for a spatial characterization of the process and it thus fails to explain how this happens along the spatial dynamics. This paper shows that, in order to explain these stylized fact of interest, we need to take into consideration local interaction, in addition to the tendency to follow the majority.

The rest of this note is organized as follows. Section 2 describes the model, Section 3 relates the models to recent literature on local interaction and Section 4 states the main results.

\section{The model}

The model formalizes in a simple way a process of public opinion formation. Each agent is to formulate an opinion that favours one of two issues at stake. We think of the latter as agreement or disagreement on one particular matter, such as abortion or capital punishment for example, or support for one of the two running candidates in an election. The dynamic process that characterizes each agent opinion formation (that we label as Private Opinion formation) is based on the idea that each agent chooses an opinion probabilistically, in a way that responds to her perception of what the opinion of the aggregate collection of individuals is. To account for the fact that the process of opinion formation is typically highly decentralized, we shall endow each agent with a spatial location and we shall assume that, in forming an opinion, an agent is influenced by the opinions held in her immediate vicinity, i.e. by the opinion held by the agents who live in her close neighbourhood. We label the resulting process of opinion formation in the aggregate population of individuals as Public Opinion formation, and we are interested in characterizing its evolution over time, as opinions are repeatedly revised, and over space, since the locally interactive nature of the process. 


\subsection{The individual process of opinion formation}

In the model there are countably many identical agents that repeatedly formulate their opinion. Agents behave in an identical manner, though, as we shall see, asymmetries might arise due to differences in the information each individual possesses.

The reduced form of each individual opinion formation process is defined as follows. We let $\{0,1\}$ be the two available opinions and, for each individual $x$, we let $p(x)$ be a statistic that describes how agent $x$ perceives other agents' opinion. We shall mostly think of $p(x)$ as the relative frequency of opinion 1s held in $x$ 's neighbourhood and will formally define it later. Given $p(x)$, we assume that individual $x$ chooses opinion 1 with probability $f(p(x)) \in[0,1]$. We shall mostly be concerned with a class threshold functions based on majority rules, where $f(p(x))=1$ if $p(x)>0.5$ (meaning that individual $x$ chooses opinion 1 if surrounded by a strict majority of neighbours doing so); $f(p(x))=0$ if $p(x)<0.5$ and if $p(x)=0.5$ individual $x$ behaves according to one of the following different specifications:

(i) $x$ chooses opinion 1 ,

(ii) $x$ keeps the opinion currently held,

(iii) $x$ chooses opinion 1 with probability 0.5 .

In case (i) behaviour is described by an explicit tie-breaking rule that does not depend on $x$ 's identity. (ii) allows for differences in the tie breaking rule used by each agent, since opinions may differ among individuals. (iii) introduces a specific form of randomization whenever a strict majority does not exist. Note that rule (iii) is symmetric in the sense that $f(p)=1-f(1-p)$, where the symmetry refers to the flip rate associated with the rule (from 0 to 1 on the l.h.s., and from 1 to 0 on the r.h.s.), while rule (i) and (ii) are not, in that a bias is introduced in favour of opinion 1 and the opinion currently held respectively.

We shall also analyze two variants of these basic behavioural rules.

The first is a noisy version of a symmetric rule and it amounts to assume:

(iv) $f(p)=(1-\delta) 1\{p>0.5\}+\delta 1\{p<0.5\}+0.51\{p=0.5\}$

where $\delta \in[0,1 / 2]$ and $1\{E\}$ is an indicator function which is equal to one if event $E$ is true.

The second is the case where $f(p)$ is linear in $p$. It amounts to assuming that each agent reproduces probabilistically the relative frequencies of opinions held in her neighbourhood and it can be given a simple interpretation in terms of pure imitative behaviour: 
(v) $f(p)=p$

Clearly, if each agent is surrounded by exactly two neighbours, rule (v) and rule (iii) are identical.

These rules describe the process of individual opinion formation on the part of a single agent at a given point in time. In order to study the process of aggregate opinion formation, we need to analyze the joint evolution of the opinions held by each agent.

\subsection{The aggregate process of opinion formation}

We have seen that, in the model, each individual revises opinion according to the opinion held by the majority of her/his neighbors. We first need to define what we mean by neighbours. In order to do this we need to define more precisely the local structure. Each individual lives at a site on a $d$-dimensional lattice, denoted by $Z^{d}$. We shall denote by $x \in Z^{d}$, the individual who lives at site $x$. The nearest neighbours of individual $x$ are those individuals living within Euclidean distance 1 from $x$, defined by $B(x) \equiv\{y:\|x-y\|=1\}$. Hereafter we shall assume that each site only looks at the $2 d$ nearest neighbors.

Each site revises her/his opinion at a random Poisson time with intensity parameter equal to one, so that the expected length of time between two consecutive opinion re-assessments, at the same site, is equal to one. The Poisson random times are independent across sites. The Poisson assumption ensures that, at any instant of time $t$, each site has a positive probability of reassessing her/his opinion, but the probability that two sites reassess their opinions simultaneously is zero. With the term configuration we mean the state of the system, at a given time, that is the collection of opinions held by the individuals at different sites at that given time. The public opinion formation process is then the evolution over time of these configurations.

We now need to introduce some notation. We denote by $\eta_{t}(x)$ the opinion held at time $t$ by the individual at site $x, x \in Z^{d}$. We say that $\eta_{t}(x)=1$ if the individual at site $x$ favors opinion 1 and $\eta_{t}(x)=0$ if the individual at site $x$ favors opinion 0 . Thus $\eta_{t}(x)$ represents the private opinion of the individual at site $x$, while $\eta_{t}=\left\{\eta_{t}(x)\right.$ for all $\left.x \in Z^{d}\right\}$ represents the public (aggregate) opinion at time $t$. It is immediate to see that $\eta_{t}$ evolves on the space $\{0,1\}^{Z^{d}}$; in fact the state of the system, or configuration, at time $t$ is the collection of the opinions $\{0,1\}$ held by the individuals at the different sites on the $d$-dimensional lattice $Z^{d}$. Also, at each time $t$ the proportion of $x$ 's 
neighbours who hold opinion 1 is given by $p\left(x, \eta_{t}\right)=(2 d)^{-1} \sum_{\{y:\|y-x\|=1\}} \eta_{t}(y)$. On the basis of this and accordingly to the rules specified before individual $x$ may decide to revise her/his opinion over time. Note that since the number of neighbours is finite the concept of majority is well defined.

All processes we study are defined over two dimensions: space (given the local specification of the model) and time (given the postulated dynamics of opinion formation). We are interested in the following questions:

Starting from a random initial condition, will the process display contagion, in the sense that, say, opinion 1 will be adopted by all individuals in the population? Will it display consensus, in the sense that, as time goes by, all agents will tend to favour the same opinion (be that 0 or 1 )? Will instead different opinions, adopted within homogenous areas of the population survive or co-exist in the long run? We shall show that whenever consensus obtains, the resulting process of clustering can be studied locally along the dynamics, by looking at the rate at which homogeneous areas grow over time.

\section{Related Literature}

Before stating the main results, it is worthwhile to nest the set-up sketched in the previous Section within the general framework used for modelling locally interactive economic environments. Over the last few years the interest in locally interactive models has grown very quickly in different areas, such as, for example, (i) coordination games (see eg. Anderlini and Ianni (1996), Blume (1993 and 1995), Ellison (1993 and 2000), Lee and Valentinyi (2000) among others), (ii) neighborhood effect and inequality (see e.g. Benabou (1996a and 1996b), Cooper (1998), Durlauf (1996a and 1996b)). The reasons for considering local rather than global interaction are multiple: local interaction may ensure a quicker a more plausible speed of convergence to the risk dominant strategy (Ellison (1993)), individual may try to optimally react only to neighbours' action because of myopic behavior or lack of information (e.g. in Blume (1995)), the cost of investment in education may be a decreasing function of neighbours investment in education (Benabou (1996a and 1996b)), general spillover and externality effects (Durlauf (1993)).

An element common to most of the papers cited above is the attempt to jointly model spatial interaction and externality effects via a random field approach, in which evolution over both time and space is analyzed. In several instances, such that choice 
between two opinions, coordination games with two actions, or decision whether to drop out of school or not, the choice is binary. A very general set up for modelling binary choices with local interaction and for deriving testable prediction has been proposed in a series of papers by Blume and Durlauf (2001), Brock and Durlauf (2000, 2001)). We shall show below that the behavioral rules (i)-(v) sketched in the previous Section can be derived as special cases of that set up.

The underlying common feature is that each agent, out of a finite or countable set, chooses an action, out of two, by maximizing an utility function that depends on three arguments: a private utility depending only on the action taken by the individual, a social interaction term capturing the utility derived by the choices of the neighbours, and an idiosyncratic shock which differs across the two actions. More formally, the individual at site $x$ is characterized by the following utility function:

$$
V\left(\eta_{t}(x)\right)=u\left(\eta_{t}(x)\right)+S\left(\eta_{t}(x), E_{x}\left(\eta_{t}(y)\right)\right)+\varepsilon\left(\eta_{t}(x)\right)
$$

where $E_{x}\left(\eta_{t}(y)\right)$ denotes the expectation of individual $x$ about the action of individual $y \in B(x), B(x)$ being the set of $x$ 's neighbours.

The first term on the right hand side of (1) captures the private utility component, while the second term quantifies the social interaction component. Different functional forms are allowed for $S$, but typically the utility derived from a choice increases with the number of neighbours making the same choice. Finally, the last term denotes a random shock component that differs across the two choices. The individual at site $x$ will choose action 1 with probability equal to:

$$
\operatorname{Pr}[\operatorname{choosing} 1]=\operatorname{Pr}\left[V\left(\eta_{t}(x)=1\right)-V\left(\eta_{t}(x)=0\right)>0\right]
$$

By choosing a specific functional form for $u, S$ and assuming that $\varepsilon\left(\eta_{t}(x)=1\right)-$ $\varepsilon\left(\eta_{t}(x)=0\right)$ has an extreme value distribution, Brock and Durlauf obtain a closed form expression for the marginal and, assuming the independence of the shocks across individuals, for the joint conditional distribution. In particular, depending on the relative strength of the individual incentive and of the social interaction terms, either a unique or multiple equilibria can arise. They also show that $\operatorname{Pr}[$ choosing 1 ] can be modelled as a generalized, locally interactive logistic model, and derive conditions for identification and testing.

Interestingly, the aggregate conditional distribution resembles the dynamics prescribed by statistical mechanics models (e.g. Ising models, as in Blume (1993)). Indeed Blume and Durlauf (2001) points out that the key difference between locally 
interactive models in statistical mechanics and locally interactive models in economics and social sciences, is that in the former the conditional distribution (or the flip rate) is postulated as primitive, while in the latter the conditional distribution is derived as a result of a (possibly myopic) optimizing behavior.

In the set up sketched above, individuals make their choices simultaneously at time $t=1,2, \ldots$, thus they need to form expectations concerning the choice taken by their neighbours. In our model, individuals have the opportunity of revising their choices at random exponential (Poisson) times, with an intensity parameter equal to one, so that the average span of time between two consecutive revisions is equal to one. These random times are identically and independently distributed across individuals. The Poisson assumption ensures that the probability of two individuals revising their choice at the same time is zero. Thus, at anytime an individual is allowed to revise, she can observe her neighbours choices, so that $E_{x}\left(\eta_{t}(y)\right)=\eta_{t}(y)$. The collection of iid, mean one, random Poisson times is denoted by $\left\{T_{x, i}\right\}_{i=1, x \in Z^{d}}^{\infty}$, and the $k$ th time the individual at site $x$ has the opportunity to revise is $\tau_{x, k}=\sum_{i=1}^{k} T_{x, i}$. Also, while the Brock-Durlauf framework does not require any particular spatial structure, here we assume that individuals live on a $d$-dimensional lattice, $Z^{d}$.

In the previous Section the conditional probabability $f(p)$ (and so the flip rates) have been postulated as primitive. We now show that they can be easily obtained as special cases of the Brock-Durlauf set-up. It is immediate to see that rules (i)-(ii), conditional on the initial distribution and on the current neighbours configuration, are purely deterministic, in the sense that, upon observing the neighbours action, each individual chooses one of the two actions with probability one. On the other hand, rules (iii),(iv),(v) are probabilistic rules, in the sense that, even conditioning on the initial distribution and on the neighbours configuration, the individual either does not have a deterministic tie-breaking rule, as in rules (iii) and (iv), or she/he randomizes over the two choices. Thus it will not be surprising that, in terms of the notation in equation (1), $\epsilon\left(\eta_{t}(x)\right)$ is a random variable degenerate on zero in cases (i) and (ii), while it is a non-degenerate random variable in cases (iii),(iv),(v).

Before we state the rules, one thing that is worth noticing is that, in all of our cases $\operatorname{Pr}\left[V\left(\eta_{t}(x)=1\right)=V\left(\eta_{t}(x)=0\right)\right]=0$. Specifically, under rules (i) and (ii) this is ruled out by our specification, while instead in rules (iii),(iv),(v) this is ensured by the fact that $\epsilon\left(\eta_{t}(x)=1\right)-\epsilon_{t}(\eta(x)=0)$ is a continuous random variable.

Using equation (1) as our reference model, the rules defined in the previous Section can be re-written as follows. 


\section{Rule (i)}

We let:

$u\left(\eta_{t}(x)\right)=\phi 1\left\{\eta_{t}(x)=1\right\}$ with $\phi \in(0,1 /(2 d))$,

$S\left(\eta_{t}(x), E_{x}\left(\eta_{t}(y)\right)\right)=\frac{1}{2 d} \sum_{y:\|y-x\|=1} 1\left\{\eta_{t}(x)=\eta_{t}(y)\right\}$ and

$\epsilon\left(\eta_{t}(x)=1\right)=\epsilon\left(\eta_{t}(x)=0\right)=0$.

As a result, equation (1) yields:

$$
V\left(\eta_{t}(x)\right)=\phi 1\left\{\eta_{t}(x)=1\right\}+\frac{1}{2 d} \sum_{y:\|y-x\|=1} 1\left\{\eta_{t}(x)=\eta_{t}(y)\right\}
$$

Since $p=\frac{1}{2 d} \sum_{y:\|y-x\|=1} 1\left\{\eta_{t}(y)=1\right\}$, we have that:

$$
\operatorname{Pr}[\text { choosing } 1]=
$$

$$
\begin{aligned}
& =\operatorname{Pr}\left[\phi+\frac{1}{2 d} \sum_{y:\|y-x\|=1} 1\left\{\eta_{t}(y)=1\right\}>\frac{1}{2 d} \sum_{y:\|y-x\|=1} 1\left\{\eta_{t}(y)=0\right\}\right] \\
& = \begin{cases}1 & \text { for } p \geq 1 / 2 \\
0 & \text { otherwise }\end{cases}
\end{aligned}
$$

It is immediate to see that by choosing a higher value for $\phi$, we can ensure that the threshold for choosing 1 is smaller than $1 / 2$, so that we may have an asymmetric threshold. As we shall see in the Section below, rule (i) ensures that in large sample all individuals will eventually coordinate on the same choice. The intuition behind this is that private incentives, homogeneous across individuals, are strong enough to outweight the effect of social interacion.

As shown in Brock and Durlauf $(2000,2001)$, the unicity or molteplicity of long run equilibria is mainly explained by the relative strength of private incentive and social interaction or conformity. In fact, the equilibrium is typically unique when the private incentive is stronger than the desire to conform. In all other rules (ii)-(v) the private incentive or utility is set equal to zero, so that we should expect multiplicity of equilibria, either in terms of consensus on either choice, or on multiple (often infinite) equilibria in which both choices co-exist in the long run. This will be indeed the case for (ii),(iii),(v).

\section{Rule (ii)}

We let:

$$
\begin{aligned}
& u\left(\eta_{t}(x)\right)=0, \\
& S\left(\eta_{t}(x), E_{x}\left(\eta_{t}(y)\right)\right)=\frac{1}{2 d+1} \sum_{y:\|y-x\| \leq 1} 1\left\{\eta_{t}(x)=\eta_{t}(y)\right\},
\end{aligned}
$$


$\epsilon\left(\eta_{t}(x)=1\right)=\epsilon\left(\eta_{t}(x)=0\right)=0$

As a result, equation (1) yields:

$$
V\left(\eta_{t}(x)\right)=\frac{1}{2 d+1} \sum_{y:\|y-x\| \leq 1} 1\left\{\eta_{t}(x)=\eta_{t}(y)\right\}
$$

Since $p=\frac{1}{2 d} \sum_{y:\|y-x\|=1} 1\left\{\eta_{t}(y)=1\right\}$, we have that:

$$
\operatorname{Pr}[\text { choosing } 1]=
$$

$=\operatorname{Pr}\left[\frac{1}{2 d+1} \sum_{y:\|y-x\| \leq 1} 1\left\{\eta_{t}(y)=1\right\}>\frac{1}{2 d+1} \sum_{y:\|y-x\| \leq 1} 1\left\{\eta_{t}(y)=0\right\}\right]$

$=\left\{\begin{array}{ll}1 & \text { for } p \geq 1 / 2 \\ 0 & \text { otherwise }\end{array}\right.$ if the individual is currently choosing 1

\section{OR}

$$
=\left\{\begin{array}{ll}
1 & \text { for } p>1 / 2 \\
0 & \text { otherwise }
\end{array} \text { if the individual is currently choosing } 0\right.
$$

In this case the individual has a private incentive, in the sense that (s)he prefers not too change opinion or action, but such private incentive is not biased in favor or against either action and it is somewhat weak with respect to the social interaction term. As a result, as we shall see in the next Section, an infinite number of configurations, where both opinion co-exist, may arise in the long run.

\section{Rule (iii)}

We let:

$u\left(\eta_{t}(x)\right)=0$,

$S\left(\eta_{t}(x), E_{x}\left(\eta_{t}(y)\right)\right)=\frac{1}{2 d} \sum_{y:\|y-x\|=1} 1\left\{\eta_{t}(x)=\eta_{t}(y)\right\}$,

$\epsilon\left(\eta_{t}(x)=1\right)-\epsilon\left(\eta_{t}(x)=0\right)$ is (independently) uniform over $\left[-\frac{1}{2 d}, \frac{1}{2 d}\right]$

As a result, equation (1) yields:

$$
V\left(\eta_{t}(x)\right)=\frac{1}{2 d} \sum_{y:\|y-x\|=1} 1\left\{\eta_{t}(x)=\eta_{t}(y)\right\}+\epsilon\left(\eta_{t}(x)\right)
$$

Note that, when $p \neq 1 / 2$ :

$$
\left|\frac{1}{2 d} \sum_{y:\|y-x\|=1} 1\left\{\eta_{t}(y)=0\right\}-\frac{1}{2 d} \sum_{y:\|y-x\|=1} 1\left\{\eta_{t}(y)=1\right\}\right| \geq \frac{1}{d},
$$


As a result, we have that:

$$
\begin{gathered}
\operatorname{Pr}[\text { choosing } 1]= \\
=\operatorname{Pr}\left[\epsilon(\eta(x)=1)-\epsilon(\eta(x)=0)>\frac{1}{2 d} \sum_{y:\|y-x\|=1}\left(1\left\{\eta_{t}(y)=0\right\}-1\left\{\eta_{t}(y)=1\right\}\right)\right] \\
= \begin{cases}1 & \text { for } p>1 / 2 \\
0 & \text { for } p<1 / 2 \\
1 / 2 & \text { for } p=1 / 2\end{cases}
\end{gathered}
$$

In this case it is the i.i.d. uniform shock over a support depending on the number of neighbours, that provides a simple randomization rule for the case of a tie.

\section{Rule (iv)}

We let:

$u\left(\eta_{t}(x)\right)=0$,

$S\left(\eta_{t}(x), E_{x}\left(\eta_{t}(y)\right)\right)=1\left\{\frac{1}{2 d} \sum_{y:\|y-x\|=1} 1\left\{\eta_{t}(x)=\eta_{t}(y)\right\}>1 / 2\right\}$

$\epsilon\left(\eta_{t}(x)=1\right)-\epsilon\left(\eta_{t}(x)=0\right)$ is (independently) uniform over $[-(1+\gamma), 1+\gamma]$ for $\gamma>0$.

As a result, equation (1) yields:

$$
V\left(\eta_{t}(x)\right)=1\left\{\frac{1}{2 d} \sum_{y:\|y-x\|=1} 1\left\{\eta_{t}(x)=\eta_{t}(y)\right\}>1 / 2\right\}+\epsilon\left(\eta_{t}(x)\right)
$$

As a result, we have that:

$$
\begin{gathered}
\operatorname{Pr}[\text { choosing } 1]= \\
=\operatorname{Pr}\left[\epsilon\left(\eta_{t}(x)=1\right)-\epsilon\left(\eta_{t}(x)=0\right)>1\left\{\frac{1}{2 d} \sum_{y:\|y-x\|=1} 1\left\{\eta_{t}(y)=0\right\}>1 / 2\right\}\right. \\
\left.\quad-1\left\{\frac{1}{2 d} \sum_{y:\|y-x\|=1} 1\left\{\eta_{t}(y)=1\right\}>1 / 2\right\}\right] \\
= \begin{cases}1-\delta & \text { for } p>1 / 2 \\
\delta & \text { for } p<1 / 2 \\
1 / 2 & \text { for } p=1 / 2\end{cases}
\end{gathered}
$$

We immediately see that the system cannot be absorbed in any state characterized by consensus over either choices. In particular we show in the Section below that in this case we shall have a unique ergodic distribution. This is explained by the fact that the random shock component, which has a "sufficiently" large support, is the 
dominant term. Indeed, for $-1<\gamma<0$, then we would obtain the same prescription as in rule (iii).

\section{Rule (v).}

We let:

$u\left(\eta_{t}(x)\right)=0$,

$S\left(\eta_{t}(x), E_{x}\left(\eta_{t}(y)\right)\right)=\frac{1}{2 d} \sum_{y:\|y-x\|=1} 1\left\{\eta_{t}(x)=\eta_{t}(y)\right\}$,

$\epsilon\left(\eta_{t}(x)=1\right)-\epsilon\left(\eta_{t}(x)=0\right)$ is (independently) uniform over $[-1,1]$

As a result, equation (1) yields:

$$
V\left(\eta_{t}(x)\right)=\frac{1}{2 d} \sum_{y:\|y-x\|=1} 1\left\{\eta_{t}(x)=\eta_{t}(y)\right\}+\epsilon\left(\eta_{t}(x)\right)
$$

and:

$$
\begin{gathered}
\operatorname{Pr}[\text { choosing } 1]= \\
=\operatorname{Pr}\left[\epsilon(\eta(x)=1)-\epsilon(\eta(x)=0)>\frac{1}{2 d} \sum_{y:\|y-x\|=1}\left(1\left\{\eta_{t}(y)=0\right\}-1\left\{\eta_{t}(y)=1\right\}\right)\right] \\
=p
\end{gathered}
$$

Hence the behaviour implied by this rule is equivalent to pure imitation: an agent samples an opinion within her neighbourhood and simply adopts it.

In the Section that follows we shall study the dynamics of the aggregate process of opinion formation when all individuals follow one (and the same) rule among those we described. As previously noticed the questions we address relate to the asymptotics of the process.

In stating our results, we shall use the following terminology. We shall say that the process shows contagion on, say opinion 1, if, starting from a random initial configuration of opinions, the process converges to a state where everybody adopts opinion 1. We shall say that the process displays consensus if, starting from a random initial configuration of opinions, the process converges to a state where everybody adopts the same opinion. Whenever consensus obtains, we shall also characterize the rate at which the implied process of clustering occurs. We shall say that the process shows co-existence if, starting from a random initial configuration of opinions, the process converges to a state where both opinions are adopted in the population. Finally, we shall say that the process is ergodic, if independently of the initial condition, the asymptotic behaviour of the process is suitably described by a unique limit distribution, that we fully characterize. 


\section{Main Results}

\subsection{Rule (i): Contagion}

First note that, under rule (i), $f(p)=1\{p \geq 1 / 2\}$ and as a result, the flip rates are asymmetric (as the flip rate from 0 to 1 is $1\{p \geq 1 / 2\}$, while the flip rate from 1 to 0 is $1-1\{p \geq 1 / 2\})$.We now show that under rule (i) the process displays contagion, in the sense that, as time grows, all individuals will tend to agree with opinion 1.

Theorem 1 Let $d=1,2$ and assume that the initial distribution is a Bernoulli product measure with parameter $\theta \in(0,1)$. If each site follows rule (i), then $\lim _{t \rightarrow \infty} P\left(\eta_{t}(x)=\right.$ $\left.1, \forall x \in Z^{d}\right)=1$

Proof. The case of $d=1$ has already been shown in Corradi and Ianni (2000, Section $5)$.

For the case of $d=2$, we proceed along the lines of Lee and Valentinyi (LV: 2000). The only relevant difference is that in their setting each site acts simultaneously at discrete time $t=1,2, \ldots$, while in our case each sites revises her position at a Poisson random time with intensity parameter equal to one, so that the probability of two sites revising their opinion simultaneously is zero. First note that any connected group of four sites favoring 1 (team in the terminology of LV) never switches to 0 . Hereafter we denote a site by its coordinates, indicating first the horizontal coordinate. Suppose $\{0,0\},\{1,0\},\{0,1\},\{1,1\}$ and $\{2,2\},\{3,2\},\{2,3\},\{3,3\}$ favor 1 , then the first time $\{2,1\}$ and $\{1,2\}$ have an opportunity of reconsidering their opinion, will favor 1 , and then the first time $\{3,1\}$ and $\{2,0\}$ have an opportunity of reconsidering their opinion, will favor 1 , and so will $\{3,0\}$ and $\{0,3\}$ eventually too. Thus the connected group $\{i, j\}$ for $i=0,1,2,3$ and $j=0,1,2,3$ eventually coordinate on 1 .

This is true for any connected group of four, each time is surrounded by two teams of 1 in two opposite coordinates. However, as each site reconsiders her opinion at random times, the time it takes for this to occur is random. In particular it may take more than three periods. Given that, in our framework equations (11) and (12) in LV do not necessarily hold. Nevertheless, we can show the desired result in a very similar manner. Consider a sequence of squares of side $4 k+1$, with $k=0,1, \ldots, N / 4$, in fact we can think at a two-dimensional lattice as a limit for $N \rightarrow \infty$ of a square of side $N$. Suppose that $\{0,0\},\{1,0\},\{0,1\},\{1,1\}$ favor 1 , and $\{i, j\},\{i+1, j\},\{i, j+$ $1\},\{i+1, j+1\}$, with $i, j=-2 k, \ldots 2 k, k=0,1, \ldots, N / 4$ are such that $\{2 k, j\},\{2 k+$ $1, j\},\{2 k, j+1\},\{2 k+1, j+1\} ;\{-2 k, j\},\{-2 k+1, j\},\{-2 k, j+1\},\{-2 k+1, j+1\}$; 
$\{i, 2 k\},\{i+1,2 k\},\{i, 2 k+1\},\{i+1,2 k+1\}$, and $\{i,-2 k\},\{i+1,-2 k\},\{i,-2 k+$ $1\},\{i+1,-2 k+1\}$ favor 1 .

That is we require that for each $k$, on each of the four faces of the square there is at least one group of 4 connected sites favoring 1 . If this is the case for each $k$, any team not coordinated on 1 (in the sense that some site in the connected group of 4 favor 0 ) is surrounded on two opposite coordinate teams favoring 1 and thus will eventually favor 1 LV (2000, p.54) show that such a configuration occurs with strictly probability $\alpha(\theta)$, for all $\theta \in(0,1)$, independent of $N$. WLG suppose that $\sqrt{N}$ is an integer and consider the $\sqrt{N}$ square of side $\sqrt{N}$, and within each of these square we can consider the configuration described above, but with $k=0, \ldots \sqrt{N} / 4$. LV $(2000$, p.56) show that the probability that at least in one of the square such configuration will exist approaches one as $N \rightarrow \infty$. The desired result then follows.

The result relies on the asymmetry of flip rates and is related to the concept of contagion proposed by Morris (2000), and analyzed by others.

\subsection{Rule (ii): Co-existence}

Note that rule (ii) implies that an agent who is not surrounded by a strict majority does not flip to a different opinion. As a result $f(p)=1\{p>1 / 2\}+1\{p=1 / 2\} \eta(x)$ and is clearly asymmetric. We now show that under rule (ii) coexistence of different opinions can arise in the long run.

Theorem 2 Let $d=1,2$ and assume the initial distribution is a Bernoulli product measure with parameter $\theta \in(0,1)$. If each site follows rule (ii), then as $t \rightarrow \infty$ infinitely many configurations, characterized by the coexistence of both opinions arise with positive probability.

Proof. For the case of $d=1$ this has been shown in Corradi and Ianni (2000, Proposition 4.6). In the case of $d=2$, note that any configuration $\{i, j\},\{i+1, j\},\{i, j+$ $1\},\{i+1, j+1\}$ favoring the same opinion is stable over time, in fact each site has at least two neighbors agreeing with her/his position. Again we can treat a two dimensional lattice, as the limit as $N \rightarrow \infty$ of a square with side $N$. We can divide this square in $\sqrt{N}$ squares of side $\sqrt{N}$. For $\theta \in(0,1)$, from Lee and Valentinyi (2000, p.56), we know that there is a strictly positive probability of having a sequence of squares of side $4 k+1, k=0,1, \ldots \sqrt{N} / 4$ characterized by the fact of having, on 
each face, at least a square of 4 connected individuals favoring the same opinion. As the arising of such configuration is independent across the different $\sqrt{N}$, we see that we can have infinitely many configurations characterized by the coexistence of two opinions.

\subsection{Rule (iii): Consensus in $\mathbf{Z}^{1}$, but Co-existence in $\mathbf{Z}^{2}$}

Recall that under rule (iii) $f(p)=1\{p>0.5\}+0.51\{p=0.5\}$ and the implied flip rates are symmetric. The result that follows shows that the asymptotics of the process depend on the dimension of the lattice: while on a one dimensional lattice consensus obtains, this is not necessarily the case on a two dimensional lattice.

Theorem 3 Let $d=1$ and assume that the initial distribution is a Bernoulli product measure with parameter $\theta \in(0,1)$. If each site follows rule (iii), then $\lim _{t \rightarrow \infty} \operatorname{Pr}\left[\eta_{t}(x)=\right.$ $\left.\eta_{t}(y)\right]=1 \quad \forall x, y \in Z^{1}$

Let $d=2$ and denote by $\mu^{0}$ and $\mu^{1}$ the measures that assign pointmass one to the configurations that show consensus, i.e. $\eta^{0}=\left\{\eta(x)=0\right.$ for all $\left.x \in Z^{2}\right\}$ and $\eta^{1}=\left\{\eta(x)=1\right.$ for all $\left.x \in Z^{2}\right\}$ respectively. If each site follows rule (iii), then the process admits invariant measures $\mu^{0}$ and $\mu^{1}$, as well as others that assign pointmass one on configurations where both opinions co-exist.

Proof. For $d=1$ the results can be proved by showing that the process $\eta_{t}$ is a linear Voter process, and this is done in Ianni and Corradi (2000) Theorem 3, part 2.

For $d=2$ the above analogy does not hold, since a linear Voter process is produced by our labelled rule (v) and rule (iii) and (v) lead to the same behaviour only if $d=1$, since, if $d=2$, an individual surrounded, say, by one neighbour choosing opinion 1 would choose opinion 1 with probability $1 / 4$ under rule (v), but with probability zero under rule (iii).

We proceed as follows. We introduce the following partial order on $\{0,1\}^{Z^{2}}$. We say that, for $\eta, \zeta \in\{0,1\}^{Z^{2}}, \eta \leq \zeta$ if $\eta(x) \leq \zeta(x)$ for all $x \in Z^{2}$. Here after $p(x, \eta)$ and $p(x, \zeta)$ denote the proportion of neighbors of $x$ favoring 1 in configurations $\eta$ and $\zeta$ respectively. Since $f$ is monotonically non decreasing, if $\eta(x)=\zeta(x)=0$ then $f(p(x, \eta)) \leq f(p(x, \zeta))$ (and, conversely, if $\eta(x)=\zeta(x)=1,1-f(p(x, \eta)) \geq$ $1-f(p(x, \zeta)))$. A system with such property is said to be attractive (see Liggett, 1985, p.134).

From Theorem 2.3 in Liggett (1985) attractivity implies that $\mu^{0}$ and $\mu^{1}$ are extreme invariant measures for the process, as stated. That these are not the only ones 
is shown by constructing the following invariant measure $\widetilde{\mu}$. Let each coordinate of $Z^{2}$ be indexed by $\{i, j\}$ for $i=0,1, \ldots$ and $j=0,1, \ldots$. Denote by $\tilde{\eta}$ the following configuration of opinions: $\eta(x)=1$ for all, and only those, $x$ such that coordinate $i$ is positive. Let $\widetilde{\mu}$ assign pointmass one on $\widetilde{\eta}$. To see that $\widetilde{\mu}$ is invariant it suffices to notice that: a) all $x$ such that $i>1$ or $i<0$ would never flip opinion (since for each of them $p=0$ or 1 ) and b) the same holds for the border between ones and zeros in the configuration, i.e. all $x$ such that $i=0,1$ (since for each of them $p=1 / 4$ or $3 / 4) . \widetilde{\eta}$ is by no means the only stable configuration where both opinions co-exists, since it can easily be checked that any configuration involving one connected band (horizontal or vertical) of width at least two of individuals choosing opinion 1, and one connected band of width at least two of individuals choosing opinion 0 , is stable under rule (iii). The difficulty in estimating the probability of one of these occurring, given a random initial condition and for a finite approximation, arises from the fact that as the number of individuals increases, the probability of each single configuration decreases, but the number of possible configurations that yield co-existence also grows. On the basis of the result of Theorem 4 that follows, we conjecture that, if $N$ is a finite approximation of $Z^{2}$ if the process is started by a Bernoulli product measure with parameter $\theta$, the probability of with which co-existence occurs, seen as a function of $\theta$ is of exactly of the same order as the probability with which consensus occurs (namely $\theta^{N}$ ).

\subsection{Rule (iv): Ergodicity}

Under rule (iv) $f(p)=(1-\delta) 1\{p>0.5\}+\delta 1\{p<0.5\}+0.51\{p=0.5\}$. The rule hence prescribes a completely mixed behaviour on the part of individuals that may adopt either opinion with positive probability. The result that follows shows that this behavioural rule implies that the system is ergodic, in the sense that starting from any initial distribution the asymptotic behaviour of the process is entirely described by a unique probability distribution, labelled as $\mu^{\delta}$. This has full support over all possible configurations and, in the one-dimensional case, for $\delta$ becoming small (and hence for rule (iv) approaching rule (iii)), it shows that the configurations that show consensus occur with higher probability than any other configuration. The result also points out different asymptotics for the two dimensional process.

Theorem 4 Let $d=1,2$ and $\delta \in\left(0, \frac{1}{2}\right]$. If each site follows rule (iv), then the process is ergodic, in the sense that starting from any initial distribution $\mu_{0}$ the process 
converges to a unique limit distribution $\mu^{\delta}$ :

$$
\lim _{t \rightarrow \infty} \mu_{t}^{\delta, \mu_{0}}=\mu^{\delta} \text { for any initial distribution } \mu_{0}
$$

and:

$$
\mu^{\delta}(\eta)=K \exp \left[\sum_{x} \sum_{\{y:\|y-x\|=1\}} \sigma^{\delta}(p(x, \eta))(2 \eta(x)-1)(2 \eta(y)-1)\right]
$$

where $K$ is such that $\sum_{\eta} \mu^{\delta}(\eta)=1$ and $\sigma^{\delta}(p)=[4 d(2 p-1)]^{-1} \log \left[\frac{f(p)}{1-f(p)}\right]$ for $p \neq 0.5$ and equal to a constant $k$ for $p=0.5$.

Furthermore, if $d=1: \lim _{\delta \rightarrow 0} \mu^{\delta}=\frac{1}{2} \mu^{0}+\frac{1}{2} \mu^{1}$

\section{Proof.}

Ergodicity is proved as follows. With analogy to the proof of Theorem 3, we can show that the process $\eta^{\delta}$ is attractive for $\delta \in\left(0, \frac{1}{2}\right]$ as assumed . For $d=1$ ergodicity can then be proved on the lines of Ianni and Corradi (2000). For $d=2$ we proceed as follows. We construct an upper and lower finite approximations of our process, and show that, since the process is attractive, these constitute an appropriate coupling, from which we infer the stated limit properties.

We shall consider a sequence of finite systems by taking $\Lambda(N)=Z^{d} \cap[-N / 2, N / 2]^{d}$ for $N=2,4, \ldots \ldots$ (where we regard the lattice $Z^{d}$ as folded to form a torus) and we let $\Lambda(N)$ be finite sets that increase to $Z^{d}$, such that $\lim _{N \rightarrow \infty} \Lambda(N)=Z^{d}$.

We define the following finite approximations of the process $\eta$ by conditioning it on the events $E_{0}=\{\eta: \eta(x)=0$ for all $x \notin \Lambda(N)\}$ and $E_{1}=\{\eta: \eta(x)=1$ for all $x \notin \Lambda(N)\}:$

$$
f_{i}^{N}(p(x, \eta))=\left\{\begin{array}{cl}
f(p(x, \eta)) & \text { if } x \in \Lambda(N) \\
i & \text { if } x \notin \Lambda(N)
\end{array}\right.
$$

We denote the processes defined by $f_{i}^{N}$ by $S_{i, N}(t)$, where $S_{i, N}(t)$ is equal to the original process for $x \in \Lambda(N)$, and characterized by all coordinates set equal to $i$ for $x \notin \Lambda(N)$. Let $\mu^{0} S_{0, N}(t)$ be the law of the process characterized by $f_{0}^{N}$ when the initial distribution is given by all 0 at time 0 and let $\mu^{1} S_{1, N}(t)$ be the law of the process characterized by $f_{1}^{N}$ when the initial distribution is given by all 1 at time 0 . As $f$ is attractive, by Theorem 2.7 in Liggett (1985), also $f_{i}^{N}$ are attractive and

$$
\mu^{0} S_{0, N}(t) \leq \mu^{\theta} S(t) \leq \mu^{1} S_{1, N}(t)
$$


for $\theta \in(0,1)$, and

$$
\begin{aligned}
\lim _{N \rightarrow \infty} \lim _{t \rightarrow \infty} \mu^{0} S_{0, N}(t) & =\lim _{t \rightarrow \infty} \mu^{0} S(t) \\
\lim _{N \rightarrow \infty} \lim _{t \rightarrow \infty} \mu^{1} S_{1, N}(t) & =\lim _{t \rightarrow \infty} \mu^{1} S(t)
\end{aligned}
$$

Now notice that the process restricted to $\Lambda(N)$ can be seen as a finite Markov chain and, since $\delta \in\left(0, \frac{1}{2}\right]$ transition probabilities among states are strictly positive. As a consequence of the convergence theorem for finite-state Markov chains, independently of the initial distribution, this process converges to its unique invariant measure. Let this invariant measure be $\mu^{\delta}$; then $\lim _{t \rightarrow \infty} \mu^{0} S_{0, N}(t)=\lim _{t \rightarrow \infty} \mu^{1} S_{1, N}=\mu^{\delta}$. As $\lim _{N \rightarrow \infty} \Lambda(N)=Z^{d}$, it follows that also

$$
\lim _{N \rightarrow \infty} \lim _{t \rightarrow \infty} \mu^{0} S_{0, N}(t)=\lim _{N \rightarrow \infty} \lim _{t \rightarrow \infty} \mu^{1} S_{1, N}(t)=\mu^{\delta}
$$

which proves the first statement.

The explicit characterization of the limit distribution relies on the fact that $f(p)$ is symmetric and on the implied reversibility properties of the process. It is a straightforward computation along the lines of Ianni and Corradi (2000), part 1 of Theorem 1. The fact that $\mu^{\delta}$ posits higher probability to configurations where coordinates tend to agree with their neighbours can easily be seen by noticing that the sum of which in equation (2) is taken over all couples of nearest neighbours and the addendum is equal to one if coordinates agree, i.e. if $\eta(x)=\eta(y)$, and is equal to minus one if they do not, i.e. if $\eta(x) \neq \eta(y)$. This amounts to saying that, if $d=1$ and, say $\eta(x)=0$, the addendum can take one of the following values:

$$
(+2)\left(-\frac{1}{4} \log \left[\frac{\delta}{1-\delta}\right]\right) ; \quad(0)(k) ; \quad(-2)\left(\frac{1}{4} \log \left[\frac{1-\delta}{\delta}\right]\right)
$$

according to whether $x$ is surrounded by two, one or zero neighbours choosing 0 , respectively. Hence, for any $\delta>0, \mu^{\delta}\left(\eta^{0}\right)=\mu^{\delta}\left(\eta^{1}\right)$ and, since $\mu^{\delta}$ is upper hemicontinuous in $\delta, \lim _{\delta \rightarrow 0} \frac{\mu^{\delta}(\eta)}{\mu^{\delta}\left(\eta^{i}\right)}=0$ for all $\eta \neq \eta^{i}$ for $i=0$, 1 . Since $\mu^{i}$ denote exactly the measures that assign pointmass one on $\eta^{i}$ for $i=0,1$, the statement follows. Note that, although the process is ergodic for any positive however small $\delta, \lim _{\delta \rightarrow 0} \mu^{\delta}$ is not.

For $d=2$, things are different, as if $\eta(x)=0$, the values that the addendum can take are:

$$
\begin{gathered}
(+4)\left(-\frac{1}{8} \log \left[\frac{\delta}{1-\delta}\right]\right) ; \quad(+2)\left(-\frac{1}{4} \log \left[\frac{\delta}{1-\delta}\right]\right) \quad(0)(k) ; \\
(-2)\left(\frac{1}{4} \log \left[\frac{1-\delta}{\delta}\right]\right) \quad(-4)\left(\frac{1}{8} \log \left[\frac{1-\delta}{\delta}\right]\right)
\end{gathered}
$$


It is still clearly true that, for any $\delta>0, \mu^{\delta}\left(\eta^{0}\right)=\mu^{\delta}\left(\eta^{1}\right)$, but these are not the only configuration for which this is so. In fact consider one of the infinitely many configurations $\widehat{\eta}$ where each $x$ is surrounded by exactly three neighbours agreeing with $x$, that is for which $p(x, \widehat{\eta})=1 / 4$ for all $x$ such that $\widehat{\eta}(x)=0$ and $p(x, \widehat{\eta})=3 / 4$ for all $x$ such that $\widehat{\eta}(x)=1$. Simple computation shows that also $\mu^{\delta}(\widehat{\eta})=\mu^{\delta}\left(\eta^{0}\right)$. As a result $\lim _{\delta \rightarrow 0} \mu^{\delta}$ is not a convex combination of the pointmasses $\mu^{0}$ and $\mu^{1}$.

\subsection{Rule (v): Clustering}

According to rule (v), $f(p)=p$, each site favors an opinion with a probability equal to the frequency with which the opinion is supported by the neighbours. This rule can be interpreted as pure imitative behavior, in fact it is as if each site draws at random one of her neighbour and favors the same position. For the case of $d=1$, note that rule (iii) and (v) prescribe the same behavior. We shall see that whenever voters follow rule (v) consensus arises, in the sense that all site will eventually agree on either of the two opinions.

Theorem 5 Let $d=1,2$. If each site follows rule (v), then we shall have consensus, that is $\lim _{t \rightarrow \infty} \operatorname{Pr}\left(\eta_{t}(x)=\eta_{t}(y), \forall x, y \in Z^{d}\right)=1$. Furthermore, if the initial distribution is a Bernoulli product measure with parameter $\theta \in(0,1)$, then $\lim _{t \rightarrow \infty} \mu_{t}=\theta \mu^{1}+(1-\theta) \mu^{0}$.

Proof. From Cox and Griffeath (1986, p.350).

Thus we should expect to observe along the dynamics the formation of larger and larger areas favoring the same candidate. We shall call cluster a connected area of sites holding the same opinion, thus in one dimension a cluster is a segment of sites of the same opinion, while in two dimension a cluster is a square of sites of the same opinion. As we shall eventually reach consensus, we should expect that the cluster size will increase over time, until all the sites in the lattice will become homogeneous. This is indeed the case, although we shall see that clusters grow at a rather different rate in the one dimensional and in the two dimensional case. Let define $C\left(\eta_{t}\right)$ the mean cluster size at the origin in $Z^{1}$, that is

$$
C\left(\eta_{t}\right)=\lim _{n \rightarrow \infty} \frac{2 n}{\# \text { clusters of } \eta_{t} \text { in }[-n, n]}
$$


and let define $B^{t}(\alpha), \alpha \in(0,1)$ the average proportion of sites favoring one in a square of side $t^{\alpha / 2}$ around the origin, that is

$$
B^{t}(\alpha)=\frac{1}{t^{\alpha}} \sum_{\|x\| \leq t^{\alpha / 2}} \eta_{t}(x)
$$

Theorem 6 Suppose that the initial distribution is a Bernoulli product measure with parameter $\theta \in(0,1)$.

(i) $d=1$. The mean cluster size $C\left(\eta_{t}\right)$ grows at rate $\sqrt{t}$, in fact as $t \rightarrow \infty$, $\frac{1}{\sqrt{t}} C\left(\eta_{t}\right) \rightarrow \frac{\sqrt{\pi}}{2 \theta(1-\theta)}$

(ii) $d=2$. As $t \rightarrow \infty B^{t}(\alpha)$, as defined in (3) will converge to either 1 or 0 , for some random value of $\alpha \in(0,1)$. Thus the mean cluster size grows at a random rate slower than $\sqrt{t}$, or in other words the random value of $\alpha$ for which $B^{t}(\alpha)$ converges to either 1 or 0 has mean strictly between 0 and 1 .

Proof. (i) From Bramson and Griffeath (1980, p.191).

(ii) From Cox and Griffeath (1986, p.358), as $t \rightarrow \infty, B^{t}($.$) converges to Y_{\log (1 / .)}$ the sense of convergence of finite dimensional distribution. $Y_{\log (1 / \alpha)}$ is a diffusion process (known in the literature as Wright-Fisher diffusion) solution to the following stochastic differential equation

$$
d Y_{\tau}=Y_{\tau}\left(1-Y_{\tau}\right) d \tau+\sqrt{Y_{\tau}\left(1-Y_{\tau}\right)} d W_{\tau}
$$

where $\tau=\log (1 / \alpha), W$ is a standard Brownian motion, and $Y_{\tau}$ takes values in $[0,1]$. Define $\tau^{*}=\left\{\inf \tau\right.$ : such that $Y_{\tau}=1$ or $\left.Y_{\tau}=0\right\} . \tau^{*}$ is a random variable called absorption time, and it denotes the first time at which the diffusion $Y_{\tau}$ will hit either the value 1 or 0 . Note that $b(y)=y(1-y)$ is the drift and $\sigma^{2}=y(1-y)$ is the variance of the Wright Fisher diffusion. As $t \rightarrow \infty, B^{t}($.$) converges to Y_{\log (1 / .)}$, if at some random time $\tau^{*} Y_{\tau}$ converges to either 0 or 1 , then there is a random value of $\alpha$, equal to $e^{-\tau^{*}}$, for which $B^{t}(\alpha)$ converges to either 0 or 1 . We shall proceed by first showing that $\tau \rightarrow \infty$ (i.e. $\alpha \rightarrow 0$ ) $Y_{\tau}$ will approach either 0 or 1 with probability one. First note that as $\alpha \rightarrow 1$, most of sites will be $t^{1 / 2}$ apart, and so $\lim _{\alpha \rightarrow 1} B^{t}(\alpha)=\theta$, the global density of 1 . As when $\alpha \rightarrow 1, \tau \rightarrow 0$, we can set $Y_{0}=\theta$. Now define

$$
I_{1}=\int_{0}^{\theta} \exp \left(-\int_{0}^{x} \frac{2 b(y)}{\sigma^{2}} d y\right) d x=\int_{0}^{\theta} \exp \left(-\int_{0}^{x} \frac{2 y(1-y)}{y(1-y)} d y\right) d x=\frac{1}{2}\left(1-e^{-2 \theta}\right)
$$

and

$$
I_{0}=\int_{\theta}^{1} \exp \left(-\int_{0}^{x} \frac{2 b(y)}{\sigma^{2}} d y\right) d x=\int_{0}^{\theta} \exp \left(-\int_{0}^{x} \frac{2 y(1-y)}{y(1-y)} d y\right) d x=\frac{1}{2}\left(e^{-2 \theta}-e^{-2}\right)
$$


Given that both $I_{1}$ and $I_{0}$ are finite, from Fudenberg and Harris (1992, p.427428) we have that as $\tau \rightarrow \infty Y_{\tau}$ approaches 1 with probability $\frac{I_{1}}{I_{1}+I_{0}}=\frac{1-e^{-2 \theta}}{1-e^{-2}}$ and 0 with probability $\frac{I_{0}}{I_{1}+I_{0}}=\frac{e^{-2 \theta}-e^{-2}}{1-e^{-2}}$. Thus as $\tau \rightarrow \infty Y_{\tau}$ will reach either 1 or 0 with probability one, and so as $\alpha \rightarrow 0$, for $t$ large, $\lim _{\alpha \rightarrow 1} B^{t}(\alpha)$ converges to either 0 or 1 with probability one. Given that, it follows that the expected hitting time say $\bar{\tau}^{*}$ at which $Y_{\tau}$ will reach either 1 or 0 is finite (see e.g. Ethier and Kurtz, 1986, p.421). So there will be an expected finite value of $\alpha$, say $\bar{\alpha} \in(0,1)$ for which $B^{t}(\alpha)$ converges to either 0 or 1.

From the proposition above we see that there is a substantial difference between the clustering process in one or in two dimensions. Indeed in the one dimensional case, homogeneous areas grow at rate $\sqrt{t}$, while in the two dimensional case homogeneous areas grow at a random rate slower than rate $\sqrt{t}$, in particular there is a lot of variability in the rate of growth of these areas. As in both cases the cluster size grows at a rate slower than $t$, clusters appear to be stationary along the dynamics.

\section{Conclusions}

This paper studies various versions of a locally interactive model of opinion formation defined over space and time, and involving a countable population of individuals. The underlying model formalizes an incentive on the part of each individual to conform to the opinion held by the majority of her neighbours, i.e. the set of other individuals who live at Euclidean distance one from her, and whose opinions are observed. Each individual may revise her opinion at random Poisson times, and when such an opportunity arises, she chooses an opinion according to a rule. We study the asymptotic behaviour of the implied aggregate process of opinion formation under different specifications of these rules.

A common feature of all the rules we study (i) - (v) is the presence of local externalities in opinion formation in the sense that each individual conforms to the opinion held by the strict majority of her neighbours (with probability one under rules (i), (ii) (iii) and (v) and with high probability under noisy rule (iv)). The rules differ in the behaviour they prescribe in cases where a strict majority does not exist. In particular, rules (iii), (iv) and (v) prescribe symmetric flip rates, while rule (i) and (ii) imply asymmetric flip rates that depend on the history of each individual under rule (ii), and are given exogenously under rule (i).

The presence of local externalities in opinion formation is what supports the two 
states where all individuals adopt exactly the same opinion (be that 0 or 1 ) as steady states of the aggregate dynamics under all deterministic rules (i), (ii) (iii) and (v). However, while under rules (i), (v) and (iii) in one dimension these two states are the only steady states of the process, under rules (ii) and (iii) in two dimensions an infinite number of states where both opinions are adopted may also be stationary. We refer to the former occurrence as consensus (in the sense that a permanent state of agreement among individuals is reached), and to the latter as co-existence (in the sense that permanent disagreement may be observed asymptotically). Under noisy rule (iv) all configurations of opinions may be observed with positive probability, as postulated by the limit distribution we characterize. An interesting feature that emerges is that while in the one dimensional specification, consensus is more 'likely' to be observed at low levels of noise, in the two dimensional specification this is no longer true, in that consensus and co-existence may occur with probabilities of the same order of magnitude.

Whenever consensus occurs, we are able to characterize the rate at which it is achieved, by describing the implied process of clustering among individuals. The interesting feature that emerges is that clusters only grow at rate $\sqrt{t}$. What this means is that if one observed the path of the process of public opinion at any finite time, one would see large clusters of individuals holding the same opinion that are almost stationary along the dynamics. Hence even when co-existence is ruled out asymptotically (because consensus obtains), one would still observe it along the path, as a persistent, yet not stationary, phenomenon.

Common feature of all the specifications of the models we study in this paper are that all individuals are identical (follow exactly the same rule) and that the locally interactive structure is symmetric (in the sense that it is a one or a two dimensional lattice). Hence the only form of heterogeneity among individuals stems from the fact that each individual only observes her neighbours' opinions. Yet, despite these underlying symmetries, the paper shows that these different specifications of the rules produce very different asymptotics for the aggregate process of opinion formation. Interesting extensions of these models involve explicitly modelling heterogeneities among individuals and warrant future research. 


\section{REFERENCES}

Anderlini, L. And A. Ianni (1996), "Path Dependence and Learning From Neighbours," Games and Economic Behavior, 13, 141-177.

Baumol, W.J. (1957), "Interactions between Successive Polling Results and Voting Intentions," Public Opinion Quarterly, 21, 318-323.

Benabou, R. (1993), "Working of a City: Location, Education and Production," Quarterly Journal of Economics, CVIII, 619-652.

Benabou, R. (1996), "Equity and Efficiency in Human Capital Investment: The Local Connection," Review of Economic Studies, CVIII, 619-652.

Blume, L.E. (1993), "The Statistical Mechanics of Strategic Interaction," Games and Economic Behaviour, 5, 387-424.

Blume, L.E. (1995), "The Statistical Mechanics of Best-Response Strategy Revision," Games and Economic Behaviour, 11, 111-145.

Blume, L.E. And S.N. Durlauf (2001), "The Interaction Based Approach to Socioeconomic Behaviour," in in "Social Dynamics, (S. Durlauf and H.P. Young eds), MIT and Brooking Institution Press.

Brock, W. and S.N. Durlauf (2000), "Interaction-Based Models," in in "Handbook of Econometrics, (V.J. Heckman and E. Learner eds), North-Holland.

Brock, W. And S.N. Durlauf (2001), "Discrete Choice with Social Interactions," Review of Economic Studies, ??, ??-??.

Bramson M. and D. Griffeath (1980), "Clustering and Dispersion Rates for Some Interacting Particle Systems on $Z^{1}$," Annals of Probability, 8, 183-213.

Corradi, V. And A. IAnni (2000), "A simple locally interactive model of ergodic and nonergodic growth," , University of Southampton, 0010.

Cox, J.T. And D. Griffeath (1986), "Diffusive clustering in the two dimensional voter model," Annals of Probability, 14, 347-370.

Crespi, I. (1997), The Public Opinion Process, L.E.A. Publishers. 
Durlauf, S.N. (1993), "Nonergodic Economic Growth," Review of Economic Studies, 60, 349-366.

Durlauf, S.N. (1996a), "A Theory of Persistent Income Inequality," Journal of Economic Growth, 1, 75-93.

Durlauf, S.N. (1996b), "Neighborhood Feedbacks, Endogeneous Stratification and Income Inequality," in in "Dynamic Disequilibrium Modelling: Proceedings of the Ninth International Symposium on Economic Theory and Econometrics, (W. Barnett, G. Gandolfo and C. Hillinger eds), Cambridge University Press.

Ethier, S.N. And T.G. KurTZ (1986), Markov Processes: Characterization and Convergence, Wiley.

Fudenberg, D. and C. Harris (1992), "Evolutionary dynamics with aggregate shocks," Journal of Economic Theory, 57, 420-439.

Eltison, G. (1993), "Learning, Local Interaction, and Coordination," Econometrica, 61, 1047-1071.

Ellison, G. (2000), "Basins of Attraction, Long-Run Stochastic Stability, and the Speed of Step-by-Step Evolution," The Review of Economic Studies, 67, 17-45.

Henschel, R.L. And W. Johnston (1987), "The emergency of bandwagon effects: a theory," The Sociological Quarterly, 28, 493-511.

IAnni, A. AND V. Corradi (2000), "Consensus, Contagion and Clustering in a space-time model of Public Opinion Formations," University of Southampton, 0009.

LeE, I.H. AND A. Valentinyi (2000), "Noisy Contagion Without Mutations," The Review of Economic Studies, 67, 47-56.

Liggett, T.M. (1985), Interacting Particle Systems, Springer-Verlag.

McAlister I and D.T. Studlar (1991), "Bandwagon, Underdog, or Projection? Opinion Polls and Electoral Choice in Britain 1979-1987," The Journal of Politics, 53, 720-741.

Morris, S. (2000), "Contagion," The Review of Economic Studies, 67, 57-68. 
Nadeau, R.E., E. Cloutier and J.H. Guay (1993), "New evidence about the existence of a bandwagon effect in the opinion formation process," International Political Science Review, 14, 203-213.

Simon, H.A. (1954), "Bandwagon and Underdog Effects and the Possibility pf Equilibrium Predictions," Public Opinion Quarterly, 18, 245-253.

Zuckerman, A.S., N.A. Valentino and E.W. Zuckerman (1994), "A structural Theory of Vote Choice: Social and Political Networks and Electoral Flows in Britain and in the United States," The Journal of Politics, 56, 1008-1033. 